\section{P151 Not as Fishy as it Seems: Promoting the Complex Message of Safe Fish Consumption for Women}

Jennifer Renner, MPH, jennifer.m.renner@healthpartners. com, HealthPartners Institute, 3311 East Old

Shakopee Road, Mailstop 23310A, Bloomington, MN 55425; Abigail S. Katz, PhD, HealthPartners Institute; Jeffrey P. Anderson, DSc; Patricia McCann MS, Minnesota Department of Health;

Jeanette Y. Ziegenfuss, PhD, HealthPartners

Institute

Objective: To empower women of childbearing age with improved materials promoting safe fish consumption.

Theory, Prior Research, Rationale: Fish consumption benefits women who are or could become pregnant; a developing fetus benefits from fatty acids in fish, but is also sensitive to mercury. The specific population (women who are or could become pregnant) and two-pronged message (eating some fish is healthy; eating too much is harmful) can promote confusion and may cause women to avoid fish altogether.

Description: Through a joint effort between an integrated regional health system and a state health department, we gathered quantitative and qualitative data through surveys and focus groups of health plan members and care group patients. We leveraged these data and the available literature to construct a more useful, clear message, and communicated it through a refined brochure and website. We address messaging complexity with simply laid-out guidelines, developed with women's input, in a brochure and mobile-responsive website, which is accessible via phone at point-of-sale to diminish the information-access barrier.

Evaluation: Our evaluation survey asked the same population for input on materials design, content refinement, message utility, and behavioral change. After viewing the materials, $91 \%$ of women understood which and how much fish to safely eat. $72 \%$ would "definitely" or "maybe" eat fish if they were pregnant/planning to become pregnant. Fish consumption increased among $9 \%$ of respondents.

Conclusions and Implications: Most exposed women understood our materials, but there was minimal change in consumption patterns. Future plans include robust materials dissemination along health plan and medical group pathways, and documentation of impact and reach to determine ideal methods of reaching the women needing this information most.

Funding: Environmental Protection Agency

\section{P152 Poor Diet Quality Across Ethnic Groups and Gender in U.S. Adults: National Health and Nutrition Examination Survey (NHANES) 2005- 2012}

Ingrid K. Richards Adams, PhD, MS, LDN, RD, LDE, Richards.3@osu.edu, The Ohio State University Medical Center, 453 West 10th Avenue, Columbus, $\mathrm{OH} 43210$; Colleen K. Spees, PhD, MEd, RDN, LD, FAND, The Ohio
State University Medical Center; Jessica L. Krok, PhD, MA; Christopher A. Taylor, PhD, RDN, LD, FAND

Objective: To examine diet quality among three ethnic groups and gender to establish concordance to the 20152020 Dietary Guidelines for Americans.

Study Design, Setting, Participants: Cross-sectional analysis of 24-hour dietary recall data of 19,043 adults (>19 years) from the 2005-2012 NHANES was conducted to assess ethnic and gender differences in diet quality.

Outcome Measures and Analysis: Nutrient and MyPlate equivalents estimates were used to compute the Healthy Eating Index-2010 scores, as well as the 12 subscales (total fruit, whole fruit, total vegetables, greens and beans, whole grains, dairy, total protein foods, seafood and plant proteins, fatty acids, refined grains, sodium and empty calories).

Results: A total of 9,448 Caucasians, 5,151 Hispanic/ Latinos and 4,444 African Americans were entered into the analysis. Overall diet quality for African Americans was significantly lower (mean: 45.28 (95\% CI): 44.5, 46.07) than Hispanic/Latinos 47.61 (46.91, 48.31), and Caucasians 48.21 (47.47, 48.94). African Americans had significantly lower scores for whole fruit, total vegetables, whole grains, and empty calories and significantly better scores for fatty acids and refined grains. Additionally, women had better overall diet quality scores across the race/ethnicity groups.

Conclusions and Implications: Overall dietary intakes across all ethnic groups and gender showed poor alignment with the recommendations of the Dietary Guidelines. Although interventions to improve diet quality are needed among all ethnic groups, a targeted approach is needed among minority subgroups of the population to break the cycle of poor dietary intake that is often associated with increased risk for chronic diseases.

Funding: None

\section{P153 Relationship of Weight-Related Eating Behaviors and Weight-Loss of Participants Enrolled in a Proprietary Weight Loss Program}

Megan Tschakert, BS, South Dakota State University; Kendra Kattelmann, PhD, RDN, LN, FAND, kendra. kattelmann@sdstate.edu, South Dakota State University, Box 2203, SWG 443, Brookings, SD 57007; Jessica Meendering, PhD, EP-C, South Dakota State University; Lacey McCormack, PhD, MPH, RD, LN, EP-C

Objective: The aim of this study is to evaluate weightrelated eating behaviors of participants with clinically significant weight loss (CSWL) in a proprietary weight loss program.

Study Design, Setting and Participants, and Intervention: A cross-sectional sample of participants $(n=1,454)$ enrolled in a proprietary weight-loss program that includes meal replacements and health coaching 
P153 (continued)

were queried for weight-related eating behaviors (using Weight Related Eating Questionnaire) of routine restraint (RR), compensatory restraint (CR), susceptibility to external cues (SEC), and emotional eating (EE) in relation to CSWL (defined as having achieved a weight loss greater than $10 \%$ of starting weight).

Measureable Outcome and Analysis: Participants were dichotomized into those with CSWL $(n=973)$ and with no CSWL $(n=481)$ the relationship between CSWL (controlling for age and sex) as the dependent variable and weight-related eating behaviors as the independent variable was assessed using logistic regression (Stata/SE 14). Results: Those with CSWL have higher odds of having RR (OR: 1.3, p<0.05) and CR (OR: 1.1, p<0.05) and lower odds of having SEC (OR: 0.7, p<0.05) and EE (OR: 0.8, p<0.05) than those without CSWL.

Conclusions and Implications: Weight-related eating behaviors of participants in proprietary meal replacement weight-loss programs who have successfully lost weight differ compared to those who have not. Knowledge of the relationship between CSWL and weight-related eating behaviors can be used by coaches to assist participants in reinforcing those behaviors that support weight loss. These results are limited to participants who self-select for proprietary meal-replacement weight-loss programs and cannot be generalized to other weight-loss or maintenance programs.

Funding: Sanford Health - South Dakota State University Collaborative Research program; SD Board of Regents R\&D Innovation program

\section{P154 Self-Reported Young Adult Male Androgen Deficiency Correlation with Stress and Sleep}

Camille M. Charlier, BS, MOT, West Virginia University; Makenzie L. Barr, BS; Sarah E. Colby, PhD, RD, University of Tennessee, Knoxville; Melissa D. Olfert, DrPH, RDN, melissa.olfert@mail.wvu.edu, West Virginia University, 333 AG SC Building G28, Morgantown, WV 26506

Objective: Androgen deficiency in males has traditionally been predominantly limited to older men aged $50+$ years. However, the increase in the prevalence of chronic diseases in young males is expending the conventional scope of hypogonadism as emerging evidence suggests that the condition is positively correlated with obesity, diabetes and cardiovascular disease. Little is currently known of the relationships between hormonal disruption, stress and sleep in young adult males This study investigates lifestyle behavior patterns in male college students, including screening for androgen deficiency.

Study Design, Setting, Participants, Intervention: Over 1,100 college freshmen across 8 U.S. universities participated in the USDA-funded GetFruved study, a project aimed at identifying and improving lifestyle behaviors.
Outcome Measures and Analysis: A sub-sample of 386 male students was surveyed. Instruments used include the Androgen Deficiency in the Aging Male Questionnaire (ADAM) to assess androgen deficiency, the Perceived Stress Scale to measure stress levels and the Pittsburgh Sleep Quality Index (PSQI) to evaluate sleep quality. Logistic Regression Analysis of the data was completed using JMP and SAS software.

Results: One hundred twenty-eight male participants met the criteria for androgen deficiency as defined by the ADAM questionnaire. Univariate logistic regression showed statistically significant effects of increased stress $(\mathrm{p}<0.0001)$ and poor sleep $(\mathrm{p}<0.0001)$ on ADAM scores. Following categorization, sleep findings were confirmed by an agreement analysis $(\mathrm{p}<0.0001)$. When modeled together, stress showed a stronger effect than sleep on ADAM scores $(\mathrm{p}<0.0001$ versus $\mathrm{p}=0.1235$ respectively).

Conclusions and Implications: In young adult men, correlation was found between androgen deficiency and both increased stress levels and poor sleep quality, with stress displaying the strongest effect.

Funding: USDA

\section{P155 Using a Grocery List is Associated with Higher Diet Quality but not BMI in Parents of School-Aged Children}

Elizabeth H. Ruder, PhD, MPH, RD, ehrihst@rit.edu, Rochester Institute of Technology, 180 Lomb Memorial Drive, SLA (78) - 1693, Rochester, NY 14623;

Barbara A. Lohse, PhD, RD, Rochester Institute of Technology; Diane C. Mitchell, MS, RD, Pennsylvania State University; Leslie Cunningham-Sabo, PhD, RD, Colorado State University

Objective: Define the association between grocery list use, diet quality and weight status among parents of school-aged children.

Study Design, Setting, Participants, Intervention: Parents $(n=356)$ of children participating in Fuel for Fun, a school-based culinary and physical activity intervention, completed online, tested surveys on BMI, socio-demographics and eating behaviors. A subset $(n=83)$ completed ASA24 dietary assessment to obtain Healthy Eating Index (HEI).

Outcome Measures and Analysis: Participants who shopped with a grocery list "most of the time" or "almost always" were dichotomized versus those responding "sometimes/seldom/do not do". Chi-square and independent samples t-tests compared differences in socio-demographics, eating behavior, HEI and BMI by grocery list use. Linear regression models measured the association between shopping with a list and HEI or BMI controlling for covariates.

Results: Parents (mean age $38.0 \pm 6.5$ y) were mostly white (90\%), female (87\%), and highly educated. BMI was overweight/obese for $42 \%$. Total HEI (scored 0-100) ranged from 22.0 - 77.4, mean $55.4 \pm 12.4 \mathrm{SD}$; grocery 